\title{
Intron retention enhances gene regulatory complexity in vertebrates
}

\author{
UIf Schmitz ${ }^{1,2}$ (D), Natalia Pinello ${ }^{1,2,3}$, Fangzhi Jia ${ }^{1,2}$, Sultan Alasmari ${ }^{4}$, William Ritchie ${ }^{5}$, Maria-Cristina Keightley ${ }^{4}$, \\ Shaniko Shini ${ }^{6}$, Graham J. Lieschke ${ }^{4}$, Justin J-L Wong ${ }^{1,2,3+}$ and John E. J. Rasko ${ }^{1,2,7,8^{*}+}$
}

\begin{abstract}
Background: While intron retention (IR) is now widely accepted as an important mechanism of mammalian gene expression control, it remains the least studied form of alternative splicing. To delineate conserved features of IR, we performed an exhaustive phylogenetic analysis in a highly purified and functionally defined cell type comprising neutrophilic granulocytes from five vertebrate species spanning 430 million years of evolution.

Results: Our RNA-sequencing-based analysis suggests that IR increases gene regulatory complexity, which is indicated by a strong anti-correlation between the number of genes affected by IR and the number of protein-coding genes in the genome of individual species. Our results confirm that IR affects many orthologous or functionally related genes in granulocytes. Further analysis uncovers new and unanticipated conserved characteristics of intron-retaining transcripts. We find that intron-retaining genes are transcriptionally co-regulated from bidirectional promoters. Intron-retaining genes have significantly longer 3' UTR sequences, with a corresponding increase in microRNA binding sites, some of which include highly conserved sequence motifs. This suggests that intron-retaining genes are highly regulated post-transcriptionally.
\end{abstract}

Conclusions: Our study provides unique insights concerning the role of IR as a robust and evolutionarily conserved mechanism of gene expression regulation. Our findings enhance our understanding of gene regulatory complexity by adding another contributor to evolutionary adaptation.

Keywords: Transcriptomic complexity, Granulocytes, Evolution, Alternative splicing, Intron retention, Gene regulation

\section{Background}

Most multi-exon genes ( 95\%) have more than one alternative splice form due to exon skipping/inclusion, alternative $3^{\prime}$ and $5^{\prime}$ splice site selection, or through intron retention (IR) [1]. Of these modes of alternative splicing, IR is unique as it does typically not contribute to proteomic diversity. IR affects transcripts from up to threequarters of multi-exonic genes in mammals, yet remains the least understood mode of alternative splicing [2-5].

IR occurs physiologically when the splicing machinery fails to excise introns from primary messenger RNA (mRNA) transcripts leading to the inclusion of premature

\footnotetext{
* Correspondence: j.rasko@centenary.org.au

${ }^{\dagger}$ Equal contributors

${ }^{1}$ Gene \& Stem Cell Therapy Program, Centenary Institute, University of Sydney, Camperdown 2050, NSW, Australia

2Sydney Medical School, University of Sydney, Camperdown 2050, NSW, Australia

Full list of author information is available at the end of the article
}

termination codons (PTCs) in most intron-retaining transcripts [6]. As a consequence, intron-retaining mRNA transcripts are susceptible to degradation via nonsensemediated decay (NMD) $[6,7]$. Thus, IR can reduce gene expression at the post-transcriptional level and thereby imposes an additional level of gene regulation. Indeed, we have shown previously that IR-triggered NMD in differentiating myeloid cells reduces the abundance of cognate RNA and proteins [3]. In this context, several studies have subsequently reported transcripts with included introns detained in the nucleus and not susceptible to NMD [2, 8-12]. Additionally, there are several alternative hypotheses concerning the biological functions of IR $[6,13]$. Intron-retaining transcripts may act as sentinel RNAs ready to be spliced and translated on demand, thereby inducing more rapid protein production than de novo transcription and translation [5, 10, 14]. Other downstream effects of IR include the synthesis of novel peptides or protein isoforms, the suppression of 
protein and/or non-coding RNA (ncRNA) synthesis and the regulation of nuclear mRNA export [2, 3, 15-17]. Normal regulation of IR is essential for physiological cell functions including differentiation capacity and aberrant IR leads to human diseases including cancer [18-21].

Retained introns are known to contain, on average, a higher density of GC nucleotides and are shorter in length compared to non-retained introns [2, 3]. We and others have demonstrated that IR is a conserved mechanism that affects functionally related genes in humans and mice $[2,3,8]$. Although IR has been shown to be conserved across several vertebrate species at a tissue level, such as in the nervous system and brain [2], a thorough analysis of IR in a highly purified and functionally defined cell type has been lacking.

In an evolutionary context, the expansion of alternative splicing has been associated with increased transcriptomic complexity [22]. Although the frequency of alternative splicing reduces with evolutionary distance from primates [23], it is not known whether this is also a characteristic of IR. We aimed to determine whether IR contributes to transcriptomic complexity, to reveal affected biological processes, and to define specific conserved features. To achieve these goals, we analyzed IR in highly purified neutrophilic granulocytes from three mammalian species, one avian and one representative of the teleost fish, i.e. in total, five vertebrate species spanning 430 million years of evolution (Table 1) [24].

Investigation of IR in a cell type with conserved function across diverse species can potentially reveal unique characteristics, which would otherwise be masked when studying mixed populations of cells [2]. Neutrophilic granulocytes offer a discrete, well-defined cell type with phylogenetically conserved functions that serve as an excellent exemplar to study mechanisms of gene expression control. They are the most abundant cells of the innate immune system and consistently exhibit potent anti-microbial defenses since before the evolutionary divergence of teleost fish [25]. Our experimental design provides a tightly controlled model that allowed us to examine the relationship between IR and transcriptomic complexity.
We demonstrate that IR affects many orthologous or functionally related genes and that intron-retaining transcripts have very similar characteristics in all species investigated. For example, we found that intron-retaining genes are transcriptionally regulated from bidirectional promoters. The strong anti-correlation between the number of genes affected by IR and the number of protein-coding genes in the genome of individual species suggests that IR provides a mechanism of enhancing transcriptomic complexity.

It is unknown whether IR acts independently of other post-transcriptional mechanisms of gene expression control. In examining the relationship between IR and microRNA (miRNA)-mediated gene regulation we found that intron-retaining genes have significantly longer 3' UTR sequences that are enriched for miRNA binding sites. Our results suggest that IR is an evolutionary wellconserved form of alternative splicing that orchestrates post-transcriptional gene expression control.

\section{Results}

The function of IR is conserved over $\mathbf{4 3 0}$ million years

We have shown previously that IR affects similar biological processes during hematopoietic differentiation in human and mouse [3]. To study the functional conservation of IR events in species spanning 430 million years, we identified IR in terminally differentiated granulocytes using the IRFinder algorithm [26] (see "Methods"). For every intron the algorithm computes the IR ratio (in the range of $0-1$ ), which is an approximation of the proportion of total transcripts that retained the given intron. More specifically, the IR ratio is the ratio of the median read coverage of the intron to that of its flanking exons. We defined a threshold for the IR ratio (IR_ratio $=0.1$ ) in order to consider only biologically meaningful cases of IR for further analysis.

Of the five species, the representative of the ray-finned fishes (zebrafish) has the lowest fraction of expressed genes that are affected by IR (7.8\%). The group of more closely related mammalian species have a similar IR abundance with $13.6 \%$ of the expressed genes affected in

Table 1 Genomic characteristics of intron-retaining mammalian and vertebrate species

\begin{tabular}{|c|c|c|c|c|c|c|c|c|c|}
\hline & Genome size (GB) & Chromosomes & pc genes & nc genes & SRNA & IncRNA & Pseudogenes & GC (\%) & $\begin{array}{l}\text { Introns } \\
\mathrm{Mb}(\%)^{\mathrm{a}}\end{array}$ \\
\hline Human & 3.5 & 46 & 20,296 & 25,173 & 7703 & 14,889 & 14,424 & 41.3 & $1512.7(52.2)$ \\
\hline Mouse & 3.4 & 40 & 22,547 & 12,583 & 5530 & 6489 & 8770 & 42.3 & $992.7(37.4)$ \\
\hline Dog & 2.3 & 78 & 19,856 & 3774 & 3348 & 426 & 950 & 41.3 & $796.6(33.3)$ \\
\hline Chicken & 1.07 & 78 & 15,508 & 1558 & 1408 & 150 & 42 & 41.9 & $403.1(39.0)$ \\
\hline Zebrafish & 1.46 & 50 & 25,642 & 6008 & 3172 & 2741 & 293 & 36.7 & $722.2(52.7)$ \\
\hline
\end{tabular}

Sources of information are indicated in the "Methods" section. Data on introns were determined using the featureBits program of the UCSC genome browser. aPercent of the genome 
mouse, $17.4 \%$ in human, and $18.6 \%$ in dog. The exception in this study is the avian representative, in which a great proportion of expressed genes (40.8\%) retained one or more introns in their mRNA transcripts (Fig. 1a). Taken together, in all five species we found a considerable fraction of expressed genes that are affected by IR (see Additional file 1).

Next, we clustered all known orthologous genes based on their IR pattern in the five species under investigation. We found gene clusters in which IR events are exclusive to one particular species. While these speciesspecific clusters represent distinct sets of genes, annotation enrichment analysis revealed that similar terms are over-represented in association with all of these gene sets, as well as those in which the IR pattern is similar in several or all species (Additional file 2: Figure S1). This suggests that IR is a global control mechanism affecting functionally conserved biological processes independent of specific effector genes. Although we note that mutually enriched terms are rather general, they differ from terms

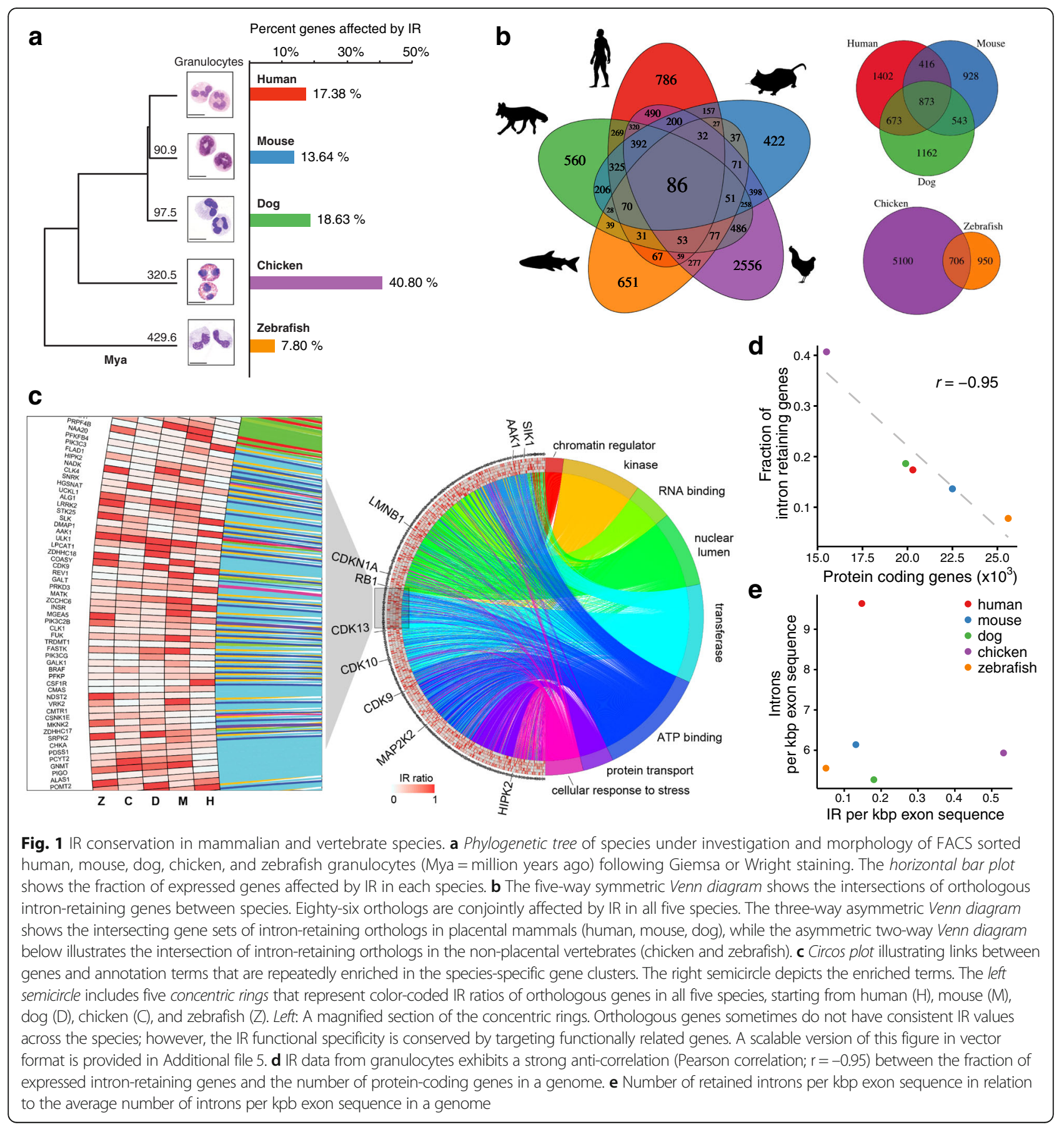


that were found enriched in other studies of IR in purified cells (Additional file 2: Table S1).

Interestingly, functionally enriched classes of genes include phosphoproteins, kinases, and genes involved in alternative splicing, i.e. groups of intron-retaining genes that realize a multitude of gene expression and protein activity control mechanisms. Consistent with this functional enrichment, Theilgaard-Mönch et al. observed an overall decline of proliferative and general cellular activity during terminal granulocytic differentiation [27]. This is plausible given that IR typically induces NMD and thereby negatively affects widespread mRNA and protein expression [3, 28]. Analogous to these observations is a recent finding that shows decreasing IR ratios associated with increasing levels of fully spliced mRNAs during $\mathrm{T}$ cell activation, to facilitate a prompt cellular response to extracellular stimuli [5].

In all species there are large numbers of intron-retaining genes with functions that are relevant to organelle lumina, most commonly the nuclear lumen. This result confirms our previous observations concerning the importance of IR in the control of granulocyte nuclear morphology [3]. The organelle lumina group includes genes that we also identified in our previous study in mouse and human granulocytes [3], e.g. $D d x 5$ (all species), Ddx3x, Lbr, Atf4, Hspa5, Ing4 (human, mouse, dog, chicken), Dnmt3a (mouse, dog, chicken), Hnrnpa2b1 (human, dog), Lmnb2 (mouse, chicken), and Lmnb1 (mouse).

Other well-conserved classes of intron-retaining gene orthologs include: kinases (e.g. Cdkn1a, Cdk9/10/13, Map2k2, Sik1), RNA-binding proteins (e.g. Upf1, $D h x 58$, $D d x 17$, Upf $3 b$ ); ATP binding proteins (e.g. $D d x 5, D d x 3 x$, Hspa5, Eif4a1, Ddx39, Atp2a3, Adrbk1); and protein transporters (Hspa5, Hsp90aa1, Hsp90ab1) (see Additional file 3).

We conclude that although specific intron-retaining genes may vary between species, they are conserved in functional clusters. Our results suggest that IR is a function-centric rather than gene-centric mechanism of coordinating gene expression (Fig. 1c). Nonetheless, there are surprisingly large numbers of gene orthologs $(n=86)$ in which IR-mediated gene regulation is conserved in the granulocytes of all five species under investigation, i.e. species that shared a common ancestral genome sequence 430 million years ago (Fig. 1b). The number of conserved intron-retaining genes is even tenfold higher among the placental mammalian species (human, mouse, dog), which share as many as 873 intron-retaining gene orthologs. The non-mammalian vertebrates share 706 intron-retaining gene orthologs (Fig. 1b). We also analyzed conservation of IR on a per-intron basis by determining orthologs of retained introns in the other species. The results suggest that although IR on a per-intron basis is less conserved, conservation is still remarkable among the mammalian species (Additional file 2: Figure S2b).
In summary, our data indicate that IR is a wellconserved mechanism of process- or function-centric gene regulation in mammalian and vertebrate species, affecting a large number of orthologous and functionally related genes.

\section{IR preserves functional complexity in species with fewer genes}

It has previously been shown that the frequency of alternative splicing events reduces with evolutionary distance from primates [23]. However, our observations of IR events in the investigated vertebrates contradict this observed trend (Fig. 1a). To determine whether retained introns, acting as gene expression control elements, preserve complexity in vertebrate species, we compared the fractions of intron-retaining genes in each species with the number of protein-coding genes in their genome. A strong anti-correlation exists between these two variables $(r=-0.95$, Pearson correlation; Fig. 1c). In contrast to other forms of alternative splicing that introduce proteomic complexity to the cell [29-32], our data indicate that IR introduces transcriptomic complexity in species with lower numbers of protein-coding genes. The most extreme example in our study is chicken in which $>40 \%$ of the 8911 expressed genes (fragments per kilobase of transcript per million mapped reads $[\mathrm{FPKM}] \geq 1$ ) in granulocytes possess retained introns, the largest fraction in all species investigated. Chicken has by far the smallest number of protein-coding genes among the five species, with a total of 15,508 . On the other end of the spectrum is zebrafish, in which $7.8 \%$ of the 768 expressed genes contain retained introns, which is a relatively small fraction in a genome that has evolved complexity via a large number of protein-coding genes $(n=25,642)$. While IR anticorrelates with the number of protein-coding genes in a genome, the correlation does not hold when we instead compare IR to the number of expressed genes. Our observations indicate transcriptional control is the dominant mechanism of gene expression control in cases where few genes are expressed (zebrafish). Post-transcriptional control of gene expression including IR is more dominant in cases where many genes are expressed (chicken). In order to reinforce this thesis, we extracted the number of transcriptional regulators (transcription factors, transcription co-factors, and chromatin remodeling factors) for each species from the AnimalTFDB database [33] and found that this number anti-correlates $(r=-0.73$, Pearson correlation) with the number of expressed genes in our samples. In zebrafish, where there are abundant transcriptional regulators, the number of expressed genes is low. We argue therefore in zebrafish that IR is not as important comparatively as it is in organisms like avian species where IR is a dominant mechanism of post-transcriptional gene expression control. Of note, intra-sample comparisons of 
gene expression values resulted in no consistent picture, showing lower median expression levels (FPKM values) of intron-retaining genes only in dog, chicken, and zebrafish (Additional file 2: Figure S3).

Despite the number of intron-retaining genes, it is also important to take into account the density of retained introns. Eukaryotic species differ substantially in their intronic density [34]. Some species haveless than 100 introns in their total genome but through spliceosomal intron evolution higher developed organisms have, on average, up to eight introns per gene [35]. In our present study humans exhibit the highest density of introns with 9.6 introns per kilobase-pair (kbp) of exonic sequences. However, chicken has the highest density of retained introns ( 0.53 retained introns/kbp exon), which is almost three times more than the species with the second highest density (dog, 0.18 retained introns/kbp exon; Fig. 1e).

Our observations suggest that IR is a phenomenon, among others, implemented to preserve transcriptomic complexity in genomes with fewer protein-coding genes. Here, complexity is effected by realizing fine-tuning of gene expression control and thereby allowing a cell to adapt to environmental changes [5]. Therefore, we propose that IR enhances gene regulatory complexity in vertebrate species.

\section{Characteristics of retained introns are well-conserved}

To define features that are conserved we compared characteristics of retained introns and their host genes in the investigated species. First, we compared the length of retained introns in granulocytes and found very similar distributions in human, mouse, dog, and chicken, where the average length of retained introns is consistently shorter than that of non-retained introns (Fig. 2a, Additional file 2: Table S2 and Figure S4). Interestingly, as the genome-specific intron length $(\mu)$ increases with genome size $\left(\mu_{\text {Chicken }}=797 \mathrm{nt} ; \mu_{\text {Zebrafish }}=1023 \mathrm{nt} ; \mu_{\text {Dog }}=\right.$ $\left.1091 \mathrm{nt} ; \mu_{\text {Mouse }}=1402 \mathrm{nt} ; \mu_{\text {Human }}=1677 \mathrm{nt}\right)$ so does the fold difference $(F D)$ between the length of non-retained introns and retained introns $\left(F D_{\text {Chicken }}=1.4 ; F D_{\text {Zebrafish }}\right.$ $\left.=2.8 ; \quad F D_{\text {Dog }}=3.1 ; \quad F D_{\text {Mouse }}=3.7 ; \quad F D_{\text {Human }}=4.9\right)$. The length of retained introns also decreases with higher IR ratios in chicken and zebrafish; however, in the mammalian species the negative trend is reversed at an IR ratio of about 0.5 in mouse and dog, and at an IR ratio of 0.7 in human (Fig. 2b). Nevertheless, the majority (more than 93\%) of retained introns examined have an IR ratio $<0.5$ (Additional file 2: Figure S5).

Others have shown previously that weak splice sites favor IR [36]. Using maximum entropy modeling [37], we confirmed this characteristic of retained introns in
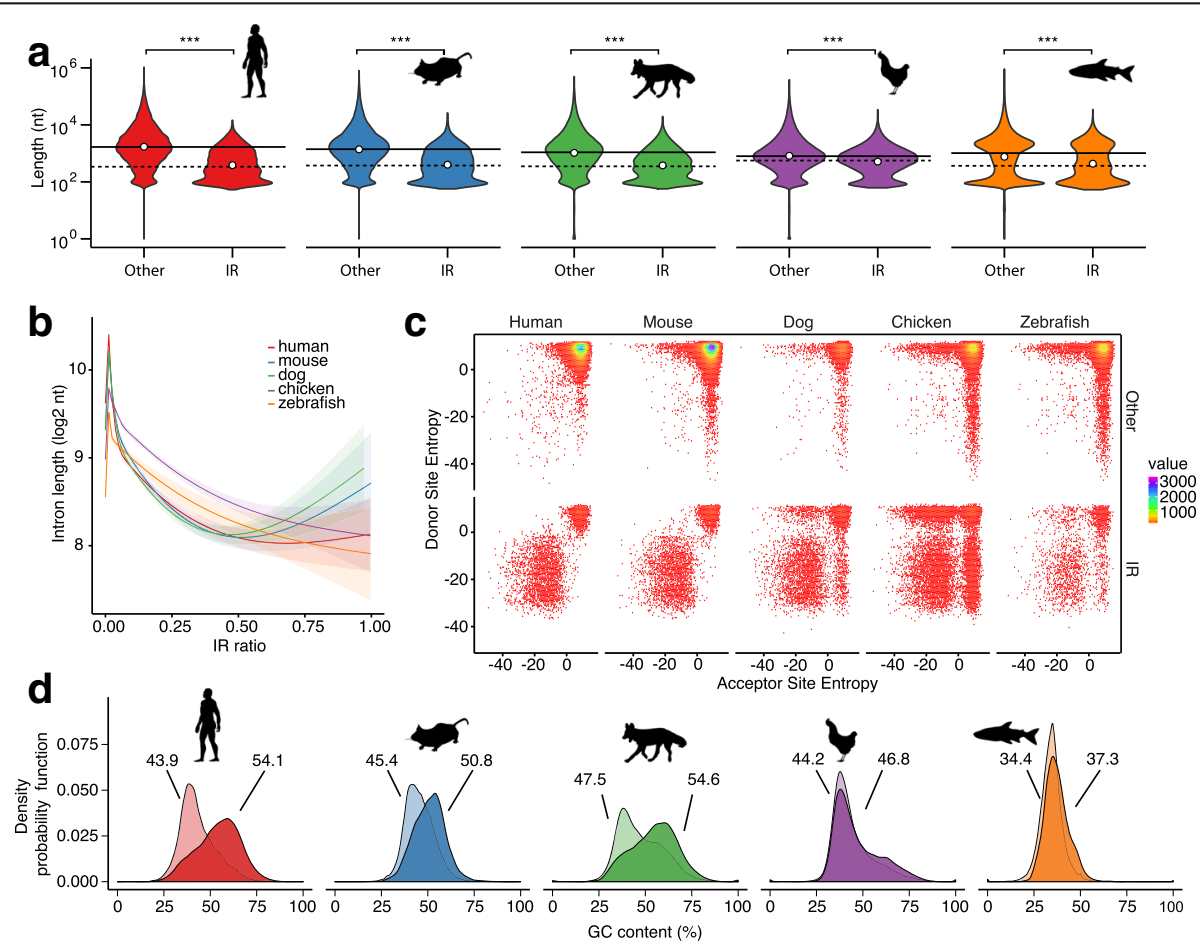

Fig. 2 Characteristics of retained introns. a Violin plots showing the log10 length distribution of non-retained (left violin in each subplot) and retained introns (right violin). Mann-Whitney $U$ test was used to determine significance, denoted by *** $(p<0.001$ ). b Generalized additive model with smoothness estimation of the intron length/IR ratio relationship. c Bivariate histograms illustrating strengths of splice site pairs (as maximum entropy) [37] of retained introns and all other introns using hexagon binning $(100 \times 100$ bins). $\mathbf{d}$ Density of the GC content in retained (dark color) and non-retained introns (light color). Numbers indicate the mean GC content 
our data and thus demonstrate that this feature is well-conserved in all species investigated (Fig. 2c). The pattern of splice site pair entropies shown in the bivariate histograms suggests that often both splice sites of retained introns are weak, thereby predisposing them for retention.

Retained introns are known to have, on average, a higher GC content compared to non-retained introns [2, 3]. We confirmed this trend in this wider spectrum of species, where the highest GC content was found in retained introns of the mammalian representatives (Fig. 2d). This reinforces our previous observations in human and mouse granulocytes as well as in murine megakaryocytes and erythrocytes [3, 4].

Intron-flanking domains expanded faster during proteomic evolution than other protein domains [38]. These mobile domains have a strong preference for phase 1 introns. Although retained introns also exhibit a phase 0 excess as observed in all introns [39], our data indicate a slight but consistent shift of the intron phase distribution away from phase 0 in retained introns in all five species (Additional file 2: Figure S6).

We have shown previously that many retained introns harbor PTCs [3]. By comparing the density of PTCs in retained vs non-retained introns, we found that retained introns incorporate slightly but significantly lower PTC densities (Additional file 2: Figure S7). The underrepresentation of PTCs in retained introns might be due to the contribution of intron-retaining isoforms that are not destined for NMD.
Intron-retaining transcripts in granulocytes are often subject to degradation via NMD mostly triggered by PTCs that facilitate detection by UPF1 triggering NMD by interacting with UPF2 and UPF3 bound to the next exonjunction complex [3, 7]. Although conditions exist in which a PTC does not lead to NMD [40], for the purpose of gene regulation via IR no more than one retained PTCcontaining intron should be required. Surprisingly, in intron-retaining genes the number of retained introns is proportional to the total number of introns in a gene (Additional file 2: Figure S8). It needs to be noted, however, that the relatively short read length $(201 \mathrm{bp})$ in Illumina RNA sequencing (RNA-seq) does not allow conclusions about the number of introns that are retained in a single transcript. Both scenarios are therefore possible: (1) different transcripts have distinct individual introns retained; or (2) transcripts harbor several retained introns. In most of the cases in human, mouse, and zebrafish, it is only one intron that is retained with a steady decrease in the frequency of cases where two or more different introns are retained (Fig. 3a). In dog, there are as many genes with three different retained introns as there are cases where only one intron is retained. Notable in this context is once again chicken, where in most of the cases three or four introns per gene are retained.

We also observed that transcripts with a larger number of introns are more prone to be affected by IR (Fig. 3b). This observation is consistent with previous studies that have identified the increase of the number

a

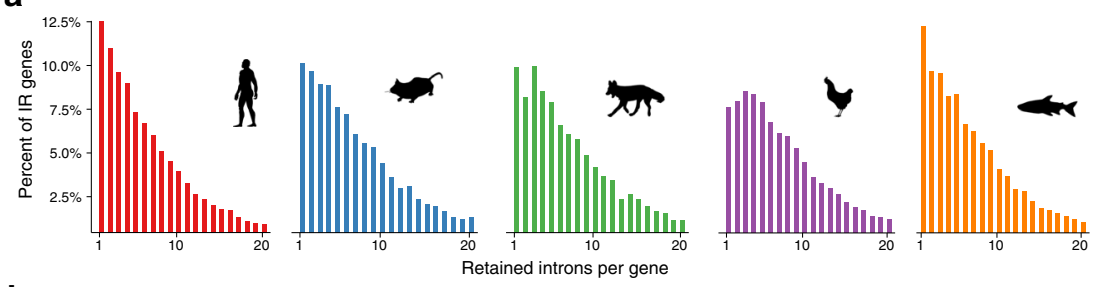

b

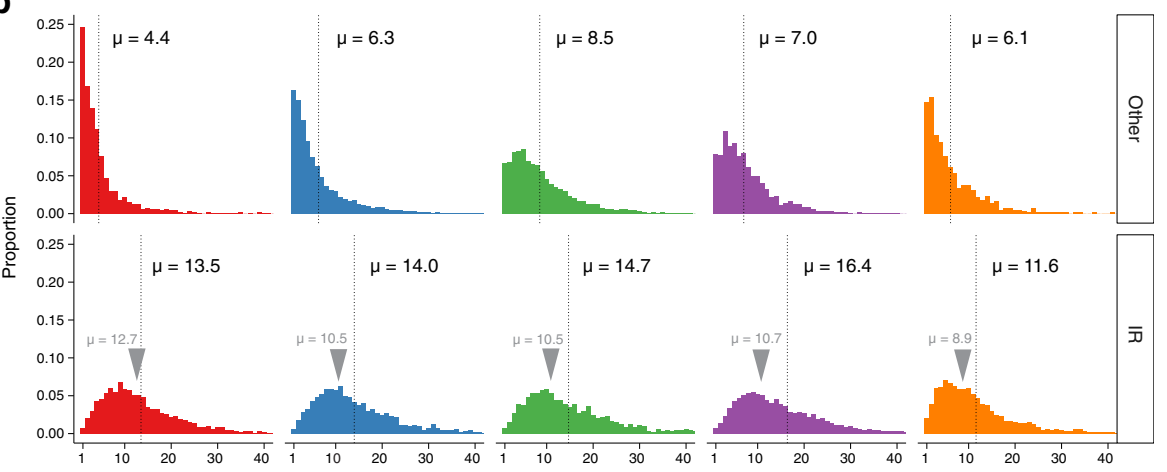

Introns

Fig. 3 Characteristics of intron-retaining genes. a Histograms of the number of retained introns in intron-retaining genes. b Distribution of intron number in transcripts without (upper panel) and with IR (lower panel) as a proportion of all transcripts. Genes that do not contain retained introns (Other) include expressed genes (FPKM > 1) only. Gray arrows above the curves indicate the average number of introns per gene in each species 
and length of introns in higher developed organisms as a way to induce genomic complexity $[41,42]$.

Bidirectional gene pairs are co-transcribed due to a mutual promoter region. It was shown previously that among adjacent human housekeeping genes there is enrichment in bidirectional gene pairs that reside in close proximity ( $<1 \mathrm{~kb}$ nt distance) [43-45]. To explore possible posttranscriptional repression via IR, we measured the distances between gene pairs that involve two intronretaining genes. Furthermore, we classified gene pairs in accordance to the published system as depicted (Fig. 4a) [46]. We consider head-to-head gene pairs as bidirectional and gene pairs with a distance $<1 \mathrm{~kb}$ as putatively co- regulated. It has been shown that many gene pairs in human and mouse share a mutual promoter region [46]. We have confirmed these findings (Additional file 2: Figure S10); however, the fraction of the gene pairs is smaller than that in the group of genes with retained introns compared to non-retained (human: $16 \%$ vs $23 \%$; mouse: $14 \%$ vs $23 \%)$. We found that the fraction of gene pairs sharing a mutual promoter is much larger in the group of genes with retained introns (human: 23\%; mouse: 23\%; Fig. 4b) compared to genes without retained introns (human: 6.4\%; mouse: 3.5\%; Additional file 2: Figure S10). Strikingly, frequencies of gene distances show an enrichment of bidirectional promoters also in dog and chicken (Fig. 4b,

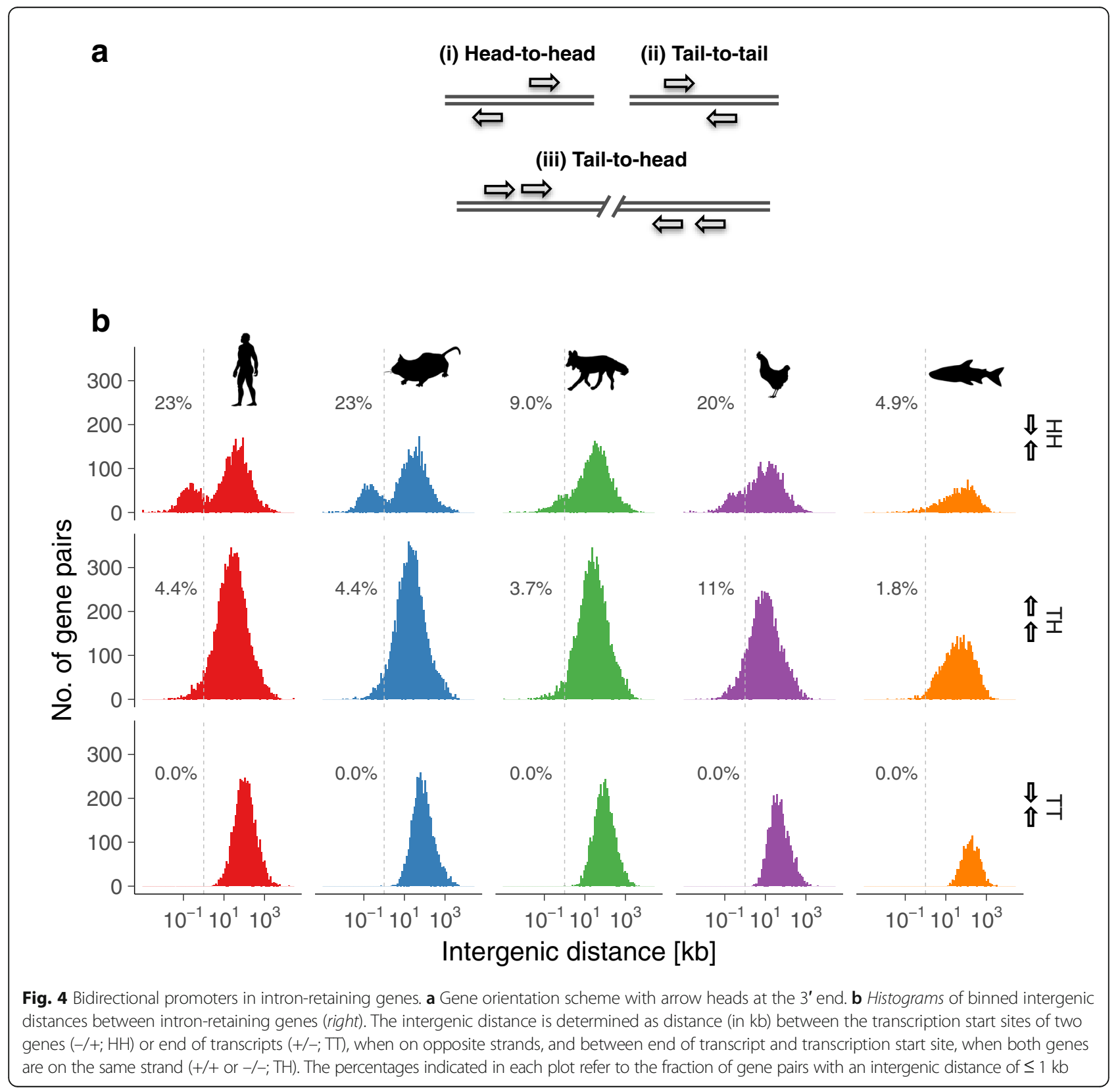


top row). The observed enrichment of bidirectional promoters could be an indication of post-transcriptionally dominated gene regulation in species/cells with a large number of expressed genes. This would explain why no enrichment has been observed in zebrafish with the lowest number of expressed genes and the largest number of transcriptional regulators in this cohort. Taken together, common regulation of transcriptional initiation or other processes occurring at the promoter may play a role in intron-retaining genes and is well-conserved.

In examining diverse species in a well-defined cell, our study corroborates previously observed characteristics of retained introns (Additional file 2: Table S3). Additionally, we demonstrated that these characteristics are wellconserved. Patterns of IR at the gene level suggest that often several different introns are retained and that transcripts with a larger number of introns have a higher probability of IR events (Additional file 2: Figure S11). These observations can be linked to previous hypotheses that the ratio of non-coding to protein-coding DNA rises as a function of transcriptomic complexity [47] and that introns fulfill many essential functions, including the regulation of gene expression, in intron-rich species [48].

\section{Intron retention complements miRNA-mediated gene regulation}

Independent observations show that retained introns predominantly reside near the $3^{\prime}$ end of a gene transcript $[2,3,18]$. We further determined the genomic distribution of retained introns to confirm previous findings and demonstrate the strong conservation of this 3' prevalence in IR (Fig. 5a). Although this pattern is consistent, in case of very long retained introns, the 3' prevalence is only preserved in zebrafish (see Additional file 2: Figure S9). Our observations with respect to the length and location of retained introns correspond with the trend of a decrease in intron length towards the 3' ends of genes in vertebrate genomes [49]. This also provides reassurance that the observed 3' prevalence of IR is neither caused by a technical nor analytical artifact. We performed gene body coverage analysis and did not find an overall bias in the mapping of any regions of genes. Thus, the $3^{\prime}$ prevalence of IR is not due to a technical bias (Additional file 2: Figure S9).

Interestingly, in human and mouse many retained introns are located in the 3' UTR (Additional file 2: Figure S12), i.e. they reside between untranslated exons and are typically spliced out during the mRNA maturation process [50]. This observation suggests the existence of novel yet unannotated 3' UTR isoforms. Because longer 3' UTR sequences may harbor additional miRNA binding sites [51], we wondered whether intron-retaining transcripts might act as competing endogenous RNAs or miRNA sponges $[6,52]$. To further investigate this possibility, we compared the 3' UTR lengths of genes with and without retained introns in their cognate mature transcripts. Surprisingly, we found that the mean 3' UTR length of intron-retaining genes is significantly longer than that of non-intron-retaining genes in all species (Fig. 5b). The difference increases in species that are evolutionarily closer to human, while in humans the median 3' UTR sequence of intron retaining genes is more than twice as long compared to the median in non-intron-retaining genes (Fig. 5b). Our evidence suggests that IR preferentially occurs in longer, more complex genes.

We predicted miRNA binding sites in the 3' UTR sequences of genes with retained introns and all other genes (not incorporating any 3' UTR intron sequences). Surprisingly, in all species the number of putative miRNA binding sites is drastically increased in genes with retained introns (Fig. 5c). The difference in miRNA binding sites in intron-retaining genes relative to nonintron-retaining genes increases with species that are evolutionarily closer to human (zebrafish: 46\%; chicken: 61\%; dog: 90\%; mouse: 126\%; and human: $256 \%$ increase). With retained introns in the 3' UTR, the number of predicted miRNA binding sites increases further by $186.3,124.1,18.1,70.4$, and 28.9 additional sites, on average, in human, mouse, dog, chicken, and zebrafish, respectively. This leads us to hypothesize that the expression of genes subject to IR is also controlled by miRNAs. MiRNA target prediction also revealed a significantly higher density of putative miRNA binding sites in sequences of retained introns compared to nonretained introns (Additional file 2: Figure S13). IR transcripts could therefore function as miRNA sponges to indirectly regulate other transcripts by modulating the available pool of miRNAs (Fig. 5e). Six examples in which retained introns may facilitate a miRNA sponge effect were derived from our previous data comparing promyelocyte against granulocyte transcriptomes in mice (Additional file 2: Figure S14; GEO accession numbers: GSE48307 [53], GSE57624 [54]). Moreover, we show based on public Ago2 HITS-CLIP data that Argonaute and possibly associated miRNAs can potentially bind to the predicted miRNA binding sites in introns (Additional file 2: Figure S15). We have also illustrated the putative effects of different miRNA expression and intron-retaining transcript levels on the expression of endogenous miRNA targets using a kinetic model based on ordinary differential equations (Additional file 2: Figure S16).

In addition, we identified significantly over-represented sequence motifs (putative miRNA seed sites) in the 3' UTR sequences of intron-retaining genes using Sylamer [55]. Interestingly, we found strong similarities among the enriched sequence motifs in mammalian species (Fig. 5d; Additional file 2: Figure S17 and Table S4) and the motifs 


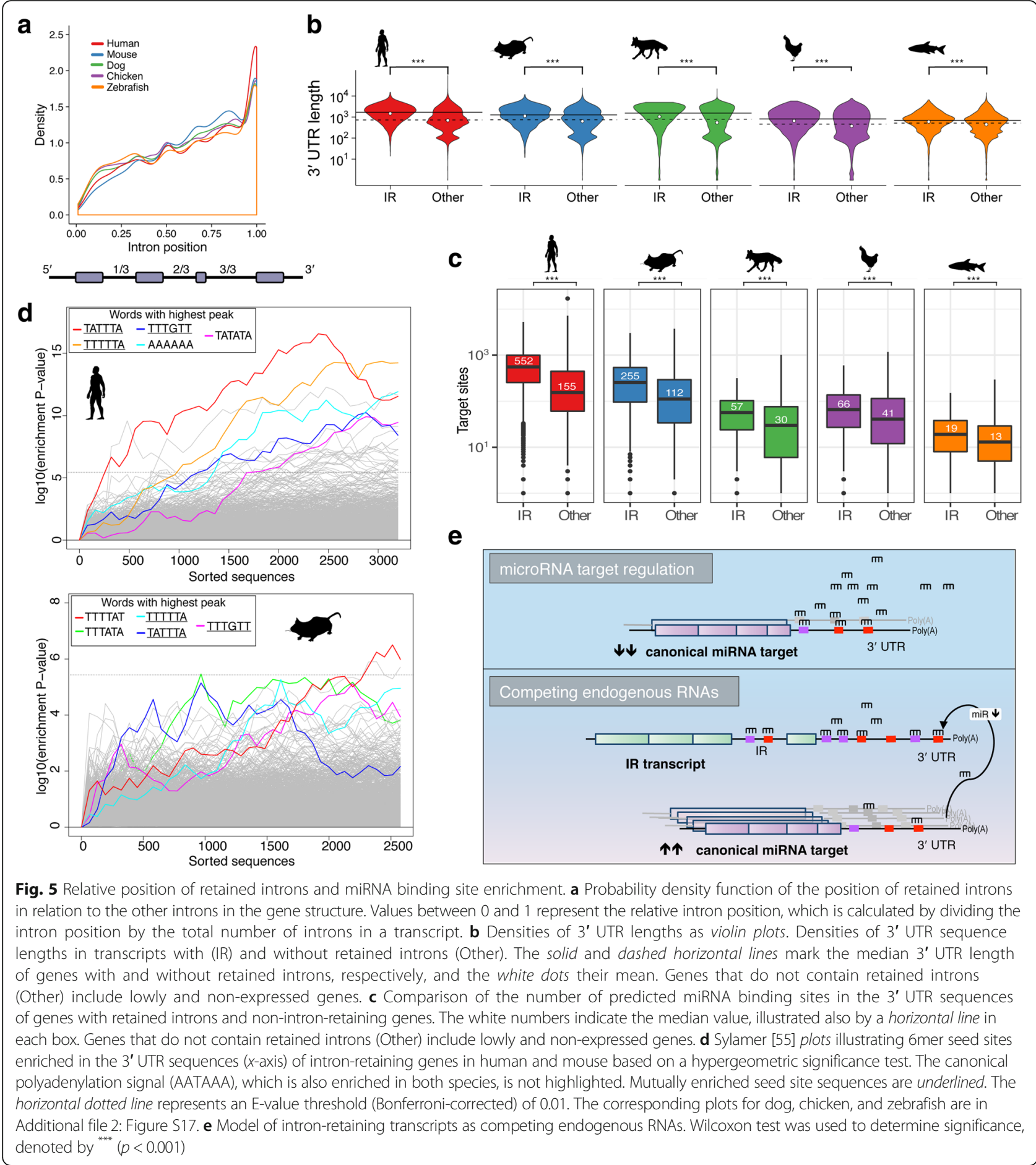

are, on average, more conserved than their flanking regions (Additional file 2: Figure S18). Taken together, these results indicate that IR is a mechanism of posttranscriptional gene regulation that complements miRNA-mediated target repression. However, given that many 3' UTR introns are retained and that intronretaining transcripts have more miRNA binding sites, they may also act as miRNA sponges as we previously proposed [6] (Fig. 5e). In support of this notion is our observation that the enriched sequence motifs are also over-represented in the 3' UTRs of genes upregulated in hematopoietic differentiation, putatively benefiting a relief from miRNAinduced expression control through intron-retaining miRNA sponge genes (Additional file 2: Figure S20). 


\section{Discussion}

Of all forms of alternative splicing, least is known about IR. One reason is that IR events are difficult to detect because cellular surveillance mechanisms like NMD can rapidly degrade transcripts with retained introns, although under certain circumstances primary transcripts can evade NMD by detention in the nucleus $[2,8,9,11,12]$. Such differences can be separated experimentally by performing nuclear:cytoplasmic fractionation, as we have previously described [3]. Furthermore, read mapping is challenging as introns are long and abundant in low-complexity regions [56]. Therefore, most transcriptomic studies focusing on alternative splicing have overlooked this type of regulation, despite its potential impact and the multitude of possible downstream effects [6].

In this in-depth phylogenetic exploration of IR in three mammalian and two non-mammalian vertebrate species, which shared a common ancestral genome 430 million years ago, we have shown that IR provides a conserved and orchestrated mechanism of posttranscriptional gene regulation. Since different subtypes of cells have vastly different mRNA splicing patterns associated with different functions, in this study we investigated IR in a highly purified and functionally defined cell type. By adopting this meticulous approach to benchmarking IR, we have extracted unique insights regarding the conservation and function of IR across species, which would otherwise likely be obscured when performing whole-tissue analysis [2, 3, 57, 58].

A surprising outcome arising from this study was the strong anti-correlation between the number of intronretaining genes and the number of protein-coding genes in a genome (Fig. 1d). This contrasts with the known increase in intron quantity with genome size, thereby facilitating increased transcriptomic complexity [34]. The false assumption that developmental complexity would be reflected by the number of protein-coding genes in a genome, referred to as the G-value paradox [59], was partially explained by alternative splicing phenomena that introduce proteomic complexity through novel protein isoforms [22, 29-31, 42]. An alternative measure suggested to account for genomic complexity is the intron density in eukaryotic organisms [30], which relates to the capacity in realizing alternative splicing events [5, 34]. By studying IR in detail, we have observed how alternative splicing utilizes introns as cis-acting regulatory gene elements to post-transcriptionally fine-tune gene expression. Therefore, we suggest that IR increases gene regulatory complexity and refer to: (1) the increased number of mRNA isoforms detectable due to IR; (2) the increased sophistication in gene expression fine-tuning (possible benefits illustrated in Additional file 2: Figure S19a/b) [60]; and (3) IR-induced complexity on a molecular network level (i.e. gene regulatory networks, metabolic networks, signaling networks) by introducing dose-dependent nonlinear dynamics (Additional file 2: Figure S19b/c) [61]. However, the ultimate function of IR can only be evaluated when the fates of intron-retaining transcripts are determined [56]. Therefore, we cannot rule out other explanations for the observed anti-correlation between the number of intron-retaining genes and the number of protein-coding genes in a genome. For example, the relative absence of IR transcripts in zebrafish granulocytes, which also express a comparatively small number of genes, could either indicate an absence of IR, or it could reflect more efficient degradation of IR transcripts than in chicken with both a high number of expressed genes and high incidence of detected IR.

Another important observation from this analysis is that intron-retaining genes harbor a larger number of miRNA binding sites (Fig. 5c), mainly generated by the presence of longer 3' UTR sequences than occur in other genes (Fig. 5b). It has been shown that the length of 3' UTRs is correlated with morphological complexity in metazoan species [62] and that gene regulation by multiple and cooperating miRNAs mediates enhanced target repression [63-65]. This indicates that IRmediated decay and miRNA-induced translational repression may be complementary mechanisms orchestrating post-transcriptional gene expression control.

Our analysis reveals that IR does not just affect many gene orthologs but also encompasses other functionally related genes, suggesting that IR is a function-centric form of gene regulation. Many of the intron-retaining genes are downregulated in differentiated granulocytes. We found, for example, 674 human intron-retaining genes to be more than twofold downregulated in the study of Theilgaard-Mönch et al., in which the authors compare gene expression profiles of human promyelocytes, myelocytes, and neutrophils [27]. The authors describe a general decline of proliferative and general cellular activity during terminal granulocytic differentiation. Here, IR seems to be a crucial regulatory factor. Downregulated genes that show IR are enriched in acetylation-related genes and splicing factors, as well as phosphoproteins and kinases and thus affect general gene expression and activity on the transcriptional, posttranscriptional, and post-translational levels, respectively (Additional file 2: Figure S21). This may also explain the G0/G1 arrest and downregulation of kinase expression observed in most end-stage differentiated neutrophil granulocytes in the study by Klausen et al. [66].

The exact mechanisms leading to IR remain to be elucidated; however, it is known that certain features increase the likelihood of an intron to be retained [36, 67]. These features are mainly composed of cis-regulatory elements marking characteristics of retained introns and their host genes $[68,69]$. In our phylogenetic study of IR 
in five species we confirmed conservation of such features including weaker splice sites, a higher GC content, and a shorter length of retained introns. Furthermore, we found that retained introns are predominantly located near the 3 ' termini of transcripts and have lower PTC densities than non-retained introns.

Most of the observations that we found conserved in all species are particularly pronounced in the avian representative. Chicken granulocytes contained the most intron-retaining genes both in number and in relation to the total number of expressed genes ( 40\%). Chicken also has the highest density of retained introns; however, their median length is larger than that observed in the other species (Fig. 2a). This is surprising because intron size correlates with genome size and bird introns are typically shorter than that of mammals [49]. This apparent paradox may have two explanations: (1) retained introns in chicken have other functions beyond regulating the expression of their host genes; or (2) they may contain more cis-regulatory elements that interact with splicing factors. Moreover, many of the chicken genes are presumably co-regulated (15\% of genes and $20 \%$ of the intronretaining genes share a mutual promoter region) and thus are more dependent on post-transcriptional gene regulation (Fig. 4, Additional file 2: Figure S10). This observation contradicts an earlier statement made by Koyanag et al. who studied the evolution of bidirectional gene pairs that share a mutual promoter region and found that enrichment in bidirectional gene pairs is only detectable in mammals and not in other eukaryotes [46].

However, the variation in IR abundance may also be explained by cell biological nuances in each species. Chicken heterophils for example are exceptional in not having a myeloperoxidase-like activity whereas this is the most abundant protein in neutrophils of the other species [70]. In general, IR calling is influenced by sequencing depth and annotation quality, i.e. the more reads and annotated genes/introns the more IR events should be detected. However, this has a negligible impact on our analysis, as the sequencing depth is comparable in all experiments. The fact that the number of retained introns detected in chicken exceeds that of all other species (including human, with the highest number of annotated introns) suggests that our results and conclusions are not biased by annotation quality.

\section{Conclusions}

In summary, our study has provided a definitive documentation of the conserved characteristics exhibited by IR in vertebrate granulocytes, including humans. We have provided new insights that support the notion of IR as an independent mechanism of gene regulation that may interfere with or complement other forms of post- transcriptional gene regulation. In IR we see a form of alternative splicing that realizes a feature contributing to gene regulatory complexity thereby facilitating organismal propensity for adaptation.

\section{Methods}

\section{Primary granulocytes}

A whole blood sample was obtained from a healthy male individual. Peripheral blood leukocytes were separated from red cells and platelets using dextran sedimentation and Ficoll density separation. Human granulocytes $(\mathrm{CD} 11 \mathrm{~b}+\mathrm{CD} 15+)$ were isolated using fluorescenceactivated cell sorting (FACS) as previously described [3].

Primary mouse granulocytes from bone marrow of male C57BL/6J mice (8-10 weeks) were purified using FACS as previously published $[3,71]$.

Peripheral blood from ten male dogs (Beagles aged 18 years) were collected from Novartis Animal Health. Dog granulocytes were purified using Percoll density separation as previously described [72], followed by FACS using a monoclonal antibody against canine neutrophils (CAD048A, Monoclonal Antibody Center, Washington State University). Purity of granulocytes was $>95 \%$ based on morphological assessment.

Chicken granulocytes were isolated from peripheral blood of six male Ross breed chickens (Gallus gallus domesticus) aged eight weeks. Following blood collection, heterophil-granulocytes were isolated as previously described [73]. Briefly, whole blood was diluted 1:1 with RPMI 1640 media (Sigma) containing 1\% methylcellulose (25 centipoises; Sigma) and centrifuged (25 g, 30 min at $4{ }^{\circ} \mathrm{C}$ ). The supernatant was transferred to a new tube, washed with calcium and magnesium-free Hank's balanced salt solution (HBSS, 1:1; Sigma), and layered onto discontinuous Ficoll-Histopaque (Sigma) gradient (specific gravity 1.077 over specific gravity 1.119). The gradients were centrifuged at $250 \mathrm{~g}$ for $60 \mathrm{~min}$ at $4{ }^{\circ} \mathrm{C}$. After centrifugation, the Histopaque layer containing the granulocytes was collected at the second interface 1.077/ 1.119 and transferred to a new Falcon tube. Cells were washed three times in RPMI 1640 media and pelleted by centrifugation at $4{ }^{\circ} \mathrm{C}, 10 \mathrm{~min}, 200 \mathrm{~g}$. The last cell pellet was resuspended in calcium and magnesium-free Hank's balanced salt solution with fetal bovine serum. Cells were further purified based on low forward and high side scatter using FACS to achieve a purity of $>95 \%$ based on morphological assessment.

Kidneys from zebrafish aged 3-6 months were dissected as previously described [74], pooled in HBSS (Sigma), homogenized, pelleted by centrifugation (250 g, $15 \mathrm{~min}$ ), and gently resuspended in $6 \mathrm{~mL} \mathrm{HBSS}$. The suspension was gently layered on $2 \mathrm{~mL}$ of lymphocyte separation medium 1078 (Mediatech; CellGro, AK, USA) in a $15-\mathrm{mL}$ Falcon tube and centrifuged (400 g, 30 
min). The resulting layer of leukocytes was removed with a $1-\mathrm{mL}$ sterile pipette and transferred to a $15-\mathrm{mL}$ tube. HBSS was added to a total volume of $4 \mathrm{~mL}$ and leukocytes collected by centrifugation (400 g, $15 \mathrm{~min}$ ). Yield assessed by hemocytometer cell counts was $1.1 \pm$ $0.6 \times 10^{6}$ cells/kidney ( $\mathrm{n}=15$ independent preparations) and samples were $88.7 \pm 6.2 \%$ (mean $\pm \mathrm{SD}$ ) granulocytes ( $\mathrm{n}=9$ random fields).

Morphological confirmation of granulocytes was performed following Giemsa (human, mouse, dog, and zebrafish) or Wright staining (chicken) of cells smeared or spun onto poly-L-lysine slides.

\section{RNA isolation and mRNA-seq}

Total RNA was isolated from granulocytes using Trizol (Invitrogen). The RNA quality was assessed using RNA 6000 Nano Chips on an Agilent Bioanalyzer (Agilent Technologies) to confirm an RNA integrity score of > 7.0. mRNA-seq was performed by Macrogen (Korea) using the Illumina Hi-Seq 2000 platform. RNA-seq libraries were prepared from $>1 \mu \mathrm{g}$ of total RNA using TruSeq RNA sample prep kit (Illumina) according to the manufacturers' instructions.

\section{Genome/gene sequences and gene structure annotations}

Whole-genome assemblies of human, mouse, dog, chicken, and zebrafish (GRCh37.75, GRCm38.78, CanFam3.1.78, Galgal4.78, Zv9.78) were downloaded from Ensembl (Release 75). Intron sequences and gene structure information were retrieved from the UCSC Table browser [75]. Data on exon phases were retrieved via the ENSEMBL BioMart interface [76].

\section{RNA-seq data analysis and identification of IR events}

Reference genome files were built and mRNA-seq reads were mapped to the respective reference genomes using STAR (Version 2.4) [77]. Details on sequencing depth and read mapping statistics for each sample are provided in Additional file 2: Table S5 and Figure S22. Gene body coverage was determined using the geneBody coverage module from the RSeQC package (v2.6.3) [78].

We used the IRFinder algorithm [26] for the detection of IR events in all known introns. IRFinder estimates the abundance of IR by computing the ratio between gene transcripts retaining an intron and the sum of all transcripts of the respective gene. We refer to this measure as the "IR ratio," while others have also used the term "percent IR" (PIR) [2]. The IR ratio is in the range of 0-1; however, we only considered introns with an IR ratio $\geq$ 0.1. We excluded introns with insufficient splicing depth ( $<4$ reads correctly crossing the splice junction) and insufficient coverage (splicing depth + trimmed mean intron depth $<10$ ). IRFinder has a built-in routine to handle confounding factors. For example, partial IR resulting from splicing inside the intron is a distinct process and was not considered. On a per-gene basis, we considered the highest observed IR ratio for any of the retained introns as the gene's IR ratio.

\section{Gene expression estimation}

Gene expression levels specified as FPKM were determined using Cufflinks [79]. FPKM values for the genes in all species are specified in Additional file 4. Ratios of intron-retaining genes to all expressed genes were determined for all genes with an FPKM value $\geq 1$.

\section{Annotation enrichment analysis}

Gene annotation enrichment was performed using the Database for Annotation, Visualization and Integrated Discovery (DAVID 6.7) [80]. In order to make the analyses comparable across species, we used the set of orthologous genes shared among all species as background. Enriched terms with a $p$ value $<0.05$ were considered significant. Most commonly enriched terms are SwissProt and Protein Information Resource keywords (SP_PIR_KEYWORDS). All enriched functional annotations for each cluster of intron-retaining orthologs illustrated in Additional file 2: Figure S1 can be found in Additional file 3. The Circos plot in Fig. 1c was generated using the $\mathrm{R}$ package GOplot [81]. We have additionally performed a functional enrichment analysis of all intronretaining genes using expressed genes as background in each species. The results are attached in Additional file 3.

\section{Gene orthologs}

Orthologs of intron-retaining genes were determined based on orthology relationships extracted from the Ensembl BioMart interface. Venn diagrams were drawn with the VennDiagram R package.

\section{miRNA target site analysis}

miRNA sequences and genomic coordinates were downloaded from the miRBase database (release 21) [82]. miRNA target site predictions were performed using miRanda v3.3a [83] with the energy threshold set to -14 $\mathrm{kcal} / \mathrm{mol}$ and requiring strict alignment in the seed region (offset positions 2-8). Seed site enrichment analysis was performed using Sylamer [55], which calculates cumulative hypergeometric $p$ values associated with small word occurrences in a sequence repository. 3' UTR sequences of intron retaining genes were tested for $6 \mathrm{mer}$ seed site enrichment using the 3' UTR sequences of all genes as background.

\section{Splice-site strength analysis}

We used the maximum entropy model prosed in Yeo and Burge [37] to estimate the strengths of donor and acceptor sites in retained and non-retained introns. 


\section{3' UTR introns}

GTF files for all species under investigation were retrieved from the ENSEMBL ftp server (ftp.ensembl.org). Introns were flagged as $3^{\prime}$ UTR-based when located in the 3' UTR of any of the transcripts of a gene.

\section{Statistical analyses}

All statistical tests were performed using the statistical programming language $\mathrm{R}$ and are specified in the main text. In Fig. 2b, we used the geom_smooth function of the $\mathrm{R}$ ggplot2 package, which uses a generalized additive model with integrated smoothness estimation to fit a curve and standard error bounds to the intron length to IR ratio relationship.

IR profiles of orthologous genes in Additional file 2: Figure S1 were quantile normalized and grouped based on $k$-means clustering (with Euclidian distance). Species-based profiles (columns) were grouped using a hierarchical clustering approach (1-pearson average linkage). Clustering and heat map visualizations were performed using GENE-E (http://www.broadinstitute.org/cancer/software/GENE-E/).

\section{Additional files}

Additional file 1: List of retained introns, their genomic coordinates, and IR ratios. (XLSX $1965 \mathrm{~kb}$ )

Additional file 2: Supplementary tables and figures. (DOCX $6927 \mathrm{~kb}$ )

Additional file 3: Sheets 1-7: All enriched functional annotations for each cluster of intron-retaining orthologs illustrated in Additional file 2: Figure S1. Sheet 8: List of homologous genes used as background. Sheets 9-13: All enriched functional annotations of all intron-retaining genes using expressed genes as background in each species. (XLSX $913 \mathrm{~kb}$ )

Additional file 4: Gene expression levels (FPKM) in all species. (XLSX $16883 \mathrm{~kb}$ )

Additional file 5: Scalable version of Fig. 1c. (PDF 8057 kb)

\section{Acknowledgements}

We thank Kim Baker and Elanco (formerly Novartis Animal Health) for providing the peripheral blood from dogs. We thank Steven Allen, Frank Kao, and Suat Dervish for FACS. The authors acknowledge the Sydney Informatics Hub and the University of Sydney's high-performance computing cluster, Artemis, for providing the high-performance computing resources that have contributed to the research results reported within this paper.

\section{Funding}

This work was supported by the National Health and Medical Research Council (Grant \#1061906 to JEJR and WR, \#1080530 to JEJR, WR, and JJW, \#1044754 and \#1086020 to GJL, \#1126306 to JJW, JEJR), Cure the Future (JEJR and US), and an anonymous foundation (JEJR). JJW and WR hold Fellowships from the Cancer Institute of NSW. US was supported by the Sydney Research Excellence Initiative. SA was supported by the King Khalid University, ABHA, KSA and the Saudi Arabian Cultural Attache in Australia. The Australian Regenerative Medicine Institute is supported by funds from the State Government of Victoria and the Australian Government.

\section{Availability of data and materials}

All RNA-seq data are available at the NCBI Sequence Read Archive (SRA project accession number: SRP092128 [84]). All other data generated during this study are included in this published article and its supplementary information files.

\section{Authors' contributions}

JEJR conceived the overall project, and supervised its execution. US, JJW, and JEJR designed the experiments. US, WR, and FJ performed statistical and bioinformatics analyses. NP, SA, SS, MCK, GJL, and JJW purified granulocytes from human, mice, dogs, chicken, and zebrafish and prepared RNA for RNAseq. US, JJW, and JEJR wrote and revised the manuscript. All authors reviewed the results and approved the manuscript.

\section{Ethics approval and consent to participate}

The human whole blood sample was obtained with ethics approval from the Human Research Ethics Committee of the Royal Prince Alfred Hospital (HREC/08/RPAH/222). The human participant has given written informed consent. Experimental methods comply with the Helsinki Declaration. Ethics approval for the primary mouse granulocytes from bone marrow of male C57BL/6J mice (8-10 weeks) was obtained from RPAH AEC Protocol no. 2013/045. Dog peripheral blood samples were collected from Novartis Animal Health with ethics approval (Protocol no. YAR-13-001 and RPAH AEC Protocol no. 2013/045). Chicken peripheral blood was collected with ethics approval (UQ AEC no. SVS/365/13/ANNANDALE and RPAH AEC Protocol no. 2013/045). The zebrafish experiments were approved by the Monash University Animal Ethics Committee (protocol MAS-2010-18).

\section{Consent for publication}

Not applicable.

\section{Competing interests}

The authors declare that they have no competing interests.

\section{Publisher's Note}

Springer Nature remains neutral with regard to jurisdictional claims in published maps and institutional affiliations.

\section{Author details}

${ }^{1}$ Gene \& Stem Cell Therapy Program, Centenary Institute, University of Sydney, Camperdown 2050, NSW, Australia. ${ }^{2}$ Sydney Medical School, University of Sydney, Camperdown 2050, NSW, Australia. ${ }^{3}$ Gene Regulation in Cancer Laboratory, Centenary Institute, University of Sydney, Camperdown 2050, NSW, Australia. ${ }^{4}$ Australian Regenerative Medicine Institute, Monash University, Clayton 3800, VIC, Australia. ${ }^{5}$ CNRS, UMR 5203, Montpellier 34094, France. ${ }^{6}$ School of Biomedical Sciences, The University of Queensland, Brisbane, QLD 4072, Australia. ${ }^{7}$ Cell and Molecular Therapies, Royal Prince Alfred Hospital, Camperdown 2050, NSW, Australia. ${ }^{8}$ Locked Bag 6, Newtown, NSW 2042, Australia.

Received: 9 October 2017 Accepted: 13 October 2017

Published online: 16 November 2017

\section{References}

1. Barash Y, Calarco JA, Gao W, Pan Q, Wang X, Shai O, Blencowe BJ, Frey BJ. Deciphering the splicing code. Nature. 2010;465:53-9.

2. Braunschweig U, Barbosa-Morais NL, Pan Q, Nachman EN, Alipanahi B, Gonatopoulos-Pournatzis T, et al. Widespread intron retention in mammals functionally tunes transcriptomes. Genome Res. 2014;24:1774-86.

3. Wong JJ, Ritchie W, Ebner OA, Selbach M, Wong JW, Huang Y, et al. Orchestrated intron retention regulates normal granulocyte differentiation. Cell. 2013;154:583-95.

4. Edwards CR, Ritchie W, Wong JJ, Schmitz U, Middleton R, An X, et al. A dynamic intron retention program in the mammalian megakaryocyte and erythrocyte lineages. Blood. 2016;127:e24-34.

5. Ni T, Yang W, Han M, Zhang Y, Shen T, Nie H, et al. Global intron retention mediated gene regulation during CD4+ T cell activation. Nucleic Acids Res. 2016;44:6817-29.

6. Wong JJ, Au AY, Ritchie W, Rasko JE. Intron retention in mRNA: No longer nonsense: Known and putative roles of intron retention in normal and disease biology. Bioessays. 2015;38:41-9.

7. Kurosaki T, Maquat LE. Nonsense-mediated mRNA decay in humans at a glance. J Cell Sci. 2016;129:461-7.

8. Boutz PL, Bhutkar A, Sharp PA. Detained introns are a novel, widespread class of post-transcriptionally spliced introns. Genes Dev. 2015;29:63-80. 
9. Pirnie SP, Osman A, Zhu Y, Carmichael GG. An Ultraconserved Element (UCE) controls homeostatic splicing of ARGLU1 mRNA. Nucleic Acids Res. 2016:45:3473-86

10. Mauger $\mathrm{O}$, Lemoine $\mathrm{F}$, Scheiffele $\mathrm{P}$. Targeted intron retention and excision for rapid gene regulation in response to neuronal activity. Neuron. 2016:92:1266-78.

11. Pimentel H, Parra M, Gee SL, Mohandas N, Pachter L, Conboy JG. A dynamic intron retention program enriched in RNA processing genes regulates gene expression during terminal erythropoiesis. Nucleic Acids Res. 2016;44:838-51.

12. Naro C, Jolly A, Di Persio S, Bielli P, Setterblad N, Alberdi AJ, et al. An orchestrated intron retention program in meiosis controls timely usage of transcripts during germ cell differentiation. Dev Cell. 2017;41:82-93.

13. Jacob AG, Smith CW. Intron retention as a component of regulated gene expression programs. Hum Genet. 2017. doi: 10.1007/s00439-017-1791-x

14. Boothby TC, Zipper RS, van der Weele CM, Wolniak SM. Removal of retained introns regulates translation in the rapidly developing gametophyte of Marsilea vestita. Dev Cell. 2013;24:517-29.

15. Bell TJ, Miyashiro KY, Sul JY, Buckley PT, Lee MT, McCullough R, et al. Intron retention facilitates splice variant diversity in calcium-activated big potassium channel populations. Proc Natl Acad Sci U S A. 2010;107:21152-7.

16. Cuenca-Bono B, Garcia-Molinero V, Pascual-Garcia P, Dopazo H, Llopis A, Vilardell J, et al. SUS1 introns are required for efficient mRNA nuclear export in yeast. Nucleic Acids Res. 2011;39:8599-611.

17. Gontijo AM, Miguela V, Whiting MF, Woodruff RC, Dominguez M. Intron retention in the Drosophila melanogaster Rieske Iron Sulphur Protein gene generated a new protein. Nat Commun. 2011;2:323.

18. Zhang Q, Li H, Jin H, Tan H, Zhang J, Sheng S. The global landscape of intron retentions in lung adenocarcinoma. BMC Med Genomics. 2014;7:15.

19. Jung $H$, Lee $D$, Lee J, Park D, Kim YJ, Park WY, et al. Intron retention is a widespread mechanism of tumor-suppressor inactivation. Nat Genet. 2015;47:1242-8.

20. Buckley PT, Lee MT, Sul JY, Miyashiro KY, Bell TJ, Fisher SA, et al. Cytoplasmic intron sequence-retaining transcripts can be dendritically targeted via ID element retrotransposons. Neuron. 2011;69:877-84.

21. Dvinge $H$, Bradley RK. Widespread intron retention diversifies most cancer transcriptomes. Genome Med. 2015;7:45

22. Chen L, Bush SJ, Tovar-Corona JM, Castillo-Morales A, Urrutia AO. Correcting for differential transcript coverage reveals a strong relationship between alternative splicing and organism complexity. Mol Biol Evol. 2014;31:1402-13.

23. Barbosa-Morais NL, Irimia M, Pan Q, Xiong HY, Gueroussov S, Lee LJ, et al. The evolutionary landscape of alternative splicing in vertebrate species. Science. 2012;338:1587-93.

24. Amores A, Catchen J, Ferrara A, Fontenot Q, Postlethwait JH. Genome evolution and meiotic maps by massively parallel DNA sequencing: spotted gar, an outgroup for the teleost genome duplication. Genetics. 2011;188:799-808.

25. Havixbeck JJ, Barreda DR. Neutrophil development, migration, and function in teleost fish. Biology. 2015;4:715-34.

26. Middleton R, Gao D, Thomas A, Singh B, Au A, Wong JJ-L, et al. IRFinder: assessing the impact of intron retention on mammalian gene expression. Genome Biol. 2017;18:51.

27. Theilgaard-Monch K, Jacobsen LC, Borup R, Rasmussen T, Bjerregaard MD, Nielsen FC, et al. The transcriptional program of terminal granulocytic differentiation. Blood. 2005;105:1785-96.

28. Jaillon O, Bouhouche K, Gout JF, Aury JM, Noel B, Saudemont B, et al. Translational control of intron splicing in eukaryotes. Nature. 2008;451:359-62

29. Blencowe BJ. Alternative splicing: new insights from global analyses. Cell. 2006;126:37-47

30. Black DL. Protein diversity from alternative splicing: a challenge for bioinformatics and post-genome biology. Cell. 2000;103:367-70.

31. Kriventseva EV, Koch I, Apweiler R, Vingron M, Bork P, Gelfand MS, et al. Increase of functional diversity by alternative splicing. Trends Genet. 2003; 19:124-8.

32. Resch A, Xing Y, Modrek B, Gorlick M, Riley R, Lee C. Assessing the impact of alternative splicing on domain interactions in the human proteome. J Proteome Res. 2004;3:76-83.

33. Zhang HM, Chen H, Liu W, Liu H, Gong J, Wang H, et al. AnimalTFDB: a comprehensive animal transcription factor database. Nucleic Acids Res. 2012;40:D144-149.

34. Rogozin IB, Carmel L, Csuros M, Koonin EV. Origin and evolution of spliceosomal introns. Biol Direct. 2012;7:11.
35. Roy SW, Gilbert W. The evolution of spliceosomal introns: patterns, puzzles and progress. Nat Rev Genet. 2006;7:211-21.

36. Ge Y, Porse BT. The functional consequences of intron retention: alternative splicing coupled to NMD as a regulator of gene expression. Bioessays. 2014;36:236-43.

37. Yeo G, Burge CB. Maximum entropy modeling of short sequence motifs with applications to RNA splicing signals. J Comput Biol. 2004;11:377-94.

38. Liu M, Walch H, Wu S, Grigoriev A. Significant expansion of exon-bordering protein domains during animal proteome evolution. Nucleic Acids Res. 2005;33:95-105.

39. Nguyen HD, Yoshihama M, Kenmochi N. Phase distribution of spliceosomal introns: implications for intron origin. BMC Evol Biol. 2006;6:69.

40. Lykke-Andersen $\mathrm{S}$, Jensen TH. Nonsense-mediated mRNA decay: an intricate machinery that shapes transcriptomes. Nat Rev Mol Cell Biol. 2015;16:665-77.

41. Lynch M, Conery JS. The origins of genome complexity. Science. 2003: 302:1401-4.

42. Taft RJ, Pheasant M, Mattick JS. The relationship between non-proteincoding DNA and eukaryotic complexity. Bioessays. 2007;29:288-99.

43. Adachi N, Lieber MR. Bidirectional gene organization: a common architectural feature of the human genome. Cell. 2002;109:807-9.

44. Takai D, Jones PA. Origins of bidirectional promoters: computational analyses of intergenic distance in the human genome. Mol Biol Evol. 2004;21:463-7.

45. Trinklein ND, Aldred SF, Hartman SJ, Schroeder DI, Otillar RP, Myers RM. An abundance of bidirectional promoters in the human genome. Genome Res. 2004;14:62-6.

46. Koyanagi KO, Hagiwara M, Itoh T, Gojobori T, Imanishi T. Comparative genomics of bidirectional gene pairs and its implications for the evolution of a transcriptional regulation system. Gene. 2005;353:169-76.

47. Mattick JS. RNA regulation: a new genetics? Nat Rev Genet. 2004;5:316-23.

48. Chorev M, Carmel L. The function of introns. Front Genet. 2012;3:55.

49. Zhang Q, Edwards SV. The evolution of intron size in amniotes: a role for powered flight? Genome Biol Evol. 2012;4:1033-43.

50. Bicknell AA, Cenik C, Chua HN, Roth FP, Moore MJ. Introns in UTRs: why we should stop ignoring them. Bioessays. 2012;34:1025-34.

51. Tan S, Guo J, Huang Q, Chen X, Li-Ling J, Li Q, et al. Retained introns increase putative microRNA targets within 3' UTRs of human mRNA. FEBS Lett. 2007;581:1081-6.

52. Salmena L, Poliseno $L$, Tay $Y$, Kats $L$, Pandolfi PP. A ceRNA hypothesis: the Rosetta Stone of a hidden RNA language? Cell. 2011;146:353-8.

53. Wong JJ, Ritchie W, Ebner OA, Selbach M, Wong JW, Huang Y, et al. Orchestrated intron retention regulates normal granulocyte differentiation. Gene Expression Omnibus. 2013. https://www.ncbi.n/m.nih.gov/geo/query/ acc.cgi?acc=GSE48307. Accessed 18 July 2016.

54. Wong JJ, Ritchie W, Gao D, Lau KA, Gonzalez M, Choudhary A, et al. Identification of nuclear-enriched miRNAs during mouse granulopoiesis. Gene Expression Omnibus. 2014. https://www.ncbi.n/m.nih.gov/geo/query/ acc.cgi?acc=GSE57624. Accessed 1 Mar 2017.

55. van Dongen S, Abreu-Goodger C, Enright AJ. Detecting microRNA binding and siRNA off-target effects from expression data. Nat Methods. 2008;5:1023-5.

56. Vanichkina DP, Schmitz U, Wong JJ, Rasko JEJ. Challenges in defining the role of intron retention in normal biology and disease. Semin Cell Dev Biol. 2017. 10.1016/j.semcdb.2017.07.030

57. Yap K, Lim ZQ, Khandelia P, Friedman B, Makeyev EV. Coordinated regulation of neuronal mRNA steady-state levels through developmentally controlled intron retention. Genes Dev. 2012;26:1209-23.

58. Welch JD, Williams LA, DiSalvo M, Brandt AT, Marayati $R$, Sims CE, et al. Selective single cell isolation for genomics using microraft arrays. Nucleic Acids Res. 2016:44:8292-301.

59. Hahn MW, Wray GA. The g-value paradox. Evol Dev. 2002;4:73-5.

60. Brawand D, Soumillon M, Necsulea A, Julien P, Csardi G, Harrigan P, et al. The evolution of gene expression levels in mammalian organs. Nature. 2011;478:343-8.

61. Tyson JJ, Chen KC, Novak B. Sniffers, buzzers, toggles and blinkers: dynamics of regulatory and signaling pathways in the cell. Curr Opin Cell Biol. 2003;15:221-31.

62. Chen $\mathrm{CY}$, Chen ST, Juan HF, Huang HC. Lengthening of $3^{\prime} U T R$ increases with morphological complexity in animal evolution. Bioinformatics. 2012;28:3178-81.

63. Schmitz U, Lai X, Winter F, Wolkenhauer O, Vera J, Gupta SK. Cooperative gene regulation by microRNA pairs and their identification using a computational workflow. Nucleic Acids Res. 2014;42:7539-52. 
64. Lai X, Bhattacharya A, Schmitz U, Kunz M, Vera J, Wolkenhauer O. A systems' biology approach to study microRNA-mediated gene regulatory networks. Biomed Res Int. 2013;2013:703849.

65. Lai X, Schmitz U, Gupta SK, Bhattacharya A, Kunz M, Wolkenhauer O, et al. Computational analysis of target hub gene repression regulated by multiple and cooperative miRNAs. Nucleic Acids Res. 2012;40:8818-34.

66. Klausen P, Bjerregaard MD, Borregaard N, Cowland JB. End-stage differentiation of neutrophil granulocytes in vivo is accompanied by upregulation of p27kip1 and down-regulation of CDK2, CDK4, and CDK6. J Leukoc Biol. 2004;75:569-78.

67. Wong JJ, Gao D, Nguyen TV, Kwok CT, van Geldermalsen M, Middleton R, et al. Intron retention is regulated by altered MeCP2-mediated splicing factor recruitment. Nat Commun. 2017:8:15134.

68. Sakabe NJ, de Souza SJ. Sequence features responsible for intron retention in human. BMC Genomics. 2007;8:59.

69. Wang Z, Burge CB. Splicing regulation: from a parts list of regulatory elements to an integrated splicing code. RNA. 2008;14:802-13.

70. Genovese KJ, He H, Swaggerty CL, Kogut MH. The avian heterophil. Dev Comp Immunol. 2013;41:334-40.

71. Wong JJ, Ritchie W, Gao D, Lau KA, Gonzalez M, Choudhary A, et al. Identification of nuclear-enriched miRNAs during mouse granulopoiesis. J Hematol Oncol. 2014;7:42.

72. Weiss DJ, Kraemer R, Schmit K. Isolation of granulocytes and mononuclear cells from the blood of dogs, cats, horses and cattle. Vet Clin Pathol. 1989;18:33-6.

73. Shini S, Shini A, Kaiser P. Cytokine and chemokine gene expression profiles in heterophils from chickens treated with corticosterone. Stress. 2010;13:185-94.

74. Lieschke GJ, Oates AC, Crowhurst MO, Ward AC, Layton JE. Morphologic and functional characterization of granulocytes and macrophages in embryonic and adult zebrafish. Blood. 2001;98:3087-96.

75. Karolchik D, Hinrichs AS, Furey TS, Roskin KM, Sugnet CW, Haussler D, et al. The UCSC Table Browser data retrieval tool. Nucleic Acids Res. 2004;32: D493-496.

76. Flicek P, Amode MR, Barrell D, Beal K, Billis K, Brent S, et al. Ensembl 2014. Nucleic Acids Res. 2014:42:D749-755.

77. Dobin A, Davis CA, Schlesinger F, Drenkow J, Zaleski C, Jha S, et al. STAR: ultrafast universal RNA-seq aligner. Bioinformatics. 2013;29:15-21.

78. Wang L, Wang S, Li W. RSeQC: quality control of RNA-seq experiments. Bioinformatics. 2012;28:2184-5.

79. Trapnell C, Williams BA, Pertea G, Mortazavi A, Kwan G, van Baren MJ, et al. Transcript assembly and quantification by RNA-Seq reveals unannotated transcripts and isoform switching during cell differentiation. Nat Biotechnol. 2010;28:511-5.

80. da Huang W, Sherman BT, Lempicki RA. Systematic and integrative analysis of large gene lists using DAVID bioinformatics resources. Nat Protoc. 2009;4:44-57.

81. Walter W, Sanchez-Cabo F, Ricote M. GOplot: an R package for visually combining expression data with functional analysis. Bioinformatics. 2015; 31:2912-4.

82. Kozomara A, Griffiths-Jones S. miRBase: annotating high confidence microRNAs using deep sequencing data. Nucleic Acids Res. 2014;42:D68-73.

83. Enright AJ, John B, Gaul U, Tuschl T, Sander C, Marks DS. MicroRNA targets in Drosophila. Genome Biol. 2003;5:R1.

84. Schmitz U, Pinello N, Jia F, Alasmari S, Ritchie W, Keightley M-C, et al. Intron retention enhances gene regulatory complexity in vertebrates. NCBI Sequence Read Archive. 2017. https://www.ncbi.nlm.nih.gov/sra/?term= SRP092128.

\section{Submit your next manuscript to BioMed Central and we will help you at every step:}

- We accept pre-submission inquiries

- Our selector tool helps you to find the most relevant journal

- We provide round the clock customer support

- Convenient online submission

- Thorough peer review

- Inclusion in PubMed and all major indexing services

- Maximum visibility for your research

Submit your manuscript at www.biomedcentral.com/submit 